\title{
Re-examining Maljkovic and Nakayama (1994): Conscious expectancy does affect the Priming of Pop-out effect
}

\author{
Brett A. Cochrane ${ }^{1}$. Jay Pratt $^{1}$ \\ Published online: 24 April 2020 \\ (C) The Psychonomic Society, Inc. 2020
}

\begin{abstract}
Maljkovic and Nakayama (Memory \& Cognition, 22(6), 657-672, 1994) observed that color singleton search performance was faster when the target and distractor colors repeated rather than switched across trials - an effect termed Priming of Pop-out (PoP). Two of the key results of this seminal study revealed that the PoP effect was not influenced by the knowledge of the probability of a target color change (Experiment 2), nor was it influenced by anticipating the upcoming target color by subvocalizing it (Experiment 4). Based on these findings they concluded that the PoP effect reflected the automatic priming due to the persistence of the target and distractor colors of the previous trial. Based on recent findings indicating that conscious expectancy may influence the PoP effect, as well as several bygone experimental practices in the original study (i.e., experimenter participants, no inferential statistics, etc.), we felt it worthwhile to evaluate whether their findings were observed when replicated in an empirically rigorous manner. Though the present study revealed that the PoP effect was robust, it was profoundly impacted by the knowledge of the probability of a target color switch (Experiment 1 ) and vocally anticipating the upcoming target color (Experiment 2). Overall, the results suggest that we should abandon the notion that the PoP effect only reflects the automatic priming of the previous target and distractor colors independent of conscious expectancy.
\end{abstract}

Keywords Priming $\cdot$ Visual search $\cdot$ Attention and memory

\section{Introduction}

It is well established that visual search speed is affected by the memory for recently observed target objects and their surrounding visual environments. A particularly notable example of this phenomenon is demonstrated in color singleton search tasks when the observer has to locate the oddball colored object among an array of homogenously colored distractor objects. When the target and distractor colors are repeated across trials of the color singleton search task, search is fast relative to when the target and distractor colors are switched (Bravo \& Nakayama, 1992). This robust effect has been termed Priming of Pop-out (PoP; Maljkovic \& Nakayama, 1994).

In a seminal paper, Maljkovic and Nakayama (1994) evaluated whether the PoP effect was affected by conscious expectancy in two experiments. First, in Experiment 2, they investigated whether the knowledge of the probability of a

Brett A. Cochrane

1 Department of Psychology, University of Toronto, Sidney Smith Hall, 100 St. George Street, Toronto, Ontario M5S 3G3, Canada target color change could influence search performance. Ken Nakayama, Vera Maljkovic, and a naïve participant performed trials of singleton search where they had to find the oddball colored diamond among two homogenously colored distractor diamonds, then indicate whether the left- or right-side corner of the target diamond was missing. Importantly, the probability that the target and distractor colors switched across trials of the search task was varied across blocks, with each of the following color-change probabilities being implemented: 0 , $.50, .70, .90$, and 1 for two participants, and $.10, .50, .70$, .90 , and 1 for the other participant. In the 0 color-change probability block, the target and distractor colors remained the same across the entire block of trials, whereas in the 1 color-change probability block the target and distractor colors switched on every trial. Maljkovic and Nakayama reasoned that if the PoP effect was due to the knowledge of the upcoming target color, then responses ought to be fastest when the target color was highly predictable (i.e., the 0 and 1 colorchange probability blocks) and slowest when it was least predictable (i.e., the .50 color-change probability block). Rather than observing this pattern of results, Maljkovic and Nakayama observed that response times increased in an approximately linear manner as the probability of a color change 
increased. They concluded that knowledge of the upcoming color did not influence the PoP effect.

Later, in Experiment 4, Maljkovic and Nakayama explored this issue further by evaluating whether an active form of conscious expectancy could influence the PoP effect. In this experiment Ken Nakayama, a graduate student, and a naïve participant performed a color singletons search task like that described previously except that the target color followed a perfectly predictable pattern (e.g., red, red, green, green, red, red, etc.). In the passive block of trials, participants performed the search task with the knowledge of the predictable target color sequence. In the active block of trials, participants were required to anticipate the upcoming target by subvocalizing its color prior to the onset of its search display. Maljkovic and Nakayama reasoned that if the PoP effect was impacted by conscious expectancy it should be attenuated in the active relative to the passive block of trials. Nonetheless, a PoP effect of similar magnitude was observed in both the active and passive blocks. Maljkovic and Nakayama concluded from this finding that an active conscious expectancy did not influence the PoP effect.

Looking back 25 years, there are reasons to think that the strong conclusions made by Maljkovic and Nakayama (1994) are not well supported by their experimental methods. First, with few participants, most of whom were experimenters, we worry that the knowledge of the theoretical underpinning of the study could have influenced responding despite the best of personal intentions. A second reason for concern is that the order in which the conditions were performed was not counterbalanced, something that has since been shown to impact the PoP effect and color singleton search more generally (Cochrane, Zhu, \& Milliken, 2018; Thomson, D'Ascenzo, \& Milliken, 2013). A third reason was that there were some unusual occurrences during Experiment 4 - the article reports that during the passive block of trials, Ken Nakayama was "chatting" with Vera Maljkovic and that the graduate student participant was listening to the radio. A final reason is that the conclusions were not based on inferential statistics. Rather, the authors relied on the traditional psychophysical technique of visually examining each participants' data. In theory this is fine but is concerning here given that not all their observations conform to the conclusions espoused by the researchers. In Experiment 2, for example, there does appear to be some deviation in the positive linear relationship between response time and the probability of a color change, suggesting that conscious expectancy does influence the PoP effect to some extent.

Recent findings cast further doubt on the conclusions of Maljkovic and Nakayama (1994). One such study investigated whether color imagery could override the PoP effect (Cochrane, Nwabuike, Thomson, \& Milliken, 2018; see also Cochrane, Zhu, \& Milliken, 2018). Participants performed a color singleton search task similar to that described previously. The critical manipulation was that participants were instructed to generate color imagery that was opposite in color to that of the previous target during the temporal interval prior to the onset of the next search display - if the previous target was red participants were to imagine green, and vice versa. The rationale was that imagining the opposite color of the previous target would put the memory representations responsible for the PoP effect in direct opposition with a visual and active form of conscious expectancy. It was observed that color imagery reversed the PoP effect such that participants were faster for target and distractor color switches (i.e., target colors that matched imagery) than for repeats (i.e., target colors that mismatched imagery). Interestingly, a different group of participants performed a version of this task where the instruction was to verbalize the opposite color of the previous target (Experiments $1 \mathrm{~b}$ and $2 \mathrm{~b}$ ). Although verbal representations of color did not influence the PoP effect to the same extent as color imagery, they did appear to attenuate the PoP effect relative to a control group where participants simply performed the search task. Indeed, the verbal group of Experiment $1 \mathrm{~b}$ produced a non-significant pattern of results that trended towards faster responding for target and distractor color switches than repeats. This verbal group finding appears to directly contrast Experiment 4 of Maljkovic and Nakayama, which showed that subvocalizing the upcoming target color did not influence the PoP effect.

Another recent study by Shurygina, Kristjánsson, Tudge, and Chetverikov (2019) also challenged the findings of Maljkovic and Nakayama (1994). In this study participants performed a singleton search task where they had to indicate the side of the missing corner on the oddball colored diamond among homogenously colored distractor diamonds. Prior to each block of trials, participants were instructed on the predictability of the upcoming target color sequence. In some blocks, the target color followed a completely predictable pattern (e.g., red, green, red, green, etc.), and in a different block, the target color followed a somewhat predictable pattern (e.g., random color, random color, red, green, random color, etc.). The researchers observed that predictable target color sequence blocks with two and five consecutive target color repeats led to faster overall responses than the somewhat predictable sequence block, which led researchers to conclude that the PoP effect was driven in part by some form of expectancy.

Given the potential shortfalls in Maljkovic and Nakayama (1994) and the recent contradictory findings (Cochrane, Nwabuike, et al., 2018; Shurygina et al., 2019), we reexamined the conclusion that conscious expectancy does not influence the PoP effect by replicating the original study. Specifically, the two experiments of the present study evaluated whether the knowledge of the probability of a color change and vocalizing the upcoming target color produced the same results as those reported by Maljkovic and Nakayama's second and fourth experiments, respectively. 
Importantly, our experiments are conceptual replications that used the same stimuli and timing that Maljkovic and Nakayama used, but counterbalanced the order of the conditions and use a statistically determined number of only naïve participants.

\section{Experiment 1}

The purpose of Experiment 1 was to replicate the procedures of Maljkovic and Nakayama's (1994) second experiment. While the basic trial procedures were replicated, this time 20 naïve participants performed the color singleton search task where they had to find the oddball colored target diamond among two homogenously colored distractor diamonds. Participants performed this search task across blocks of trials that varied the probability of a target color switch (i.e., $0, .25$, $.50, .75$, and 1$).{ }^{1}$ If the knowledge of the upcoming target color influences the PoP effect, response time should not simply increase with the probability of a target color change. However, if this knowledge does not impact the PoP effect, we expect to find a positive linear relationship between response time and the probability of a target color change.

\section{Method}

Participants Twenty undergraduates at the University of Toronto participated in exchange for course credit (16 female, $\mathrm{M}_{\mathrm{age}}=$ 18.2 years). All participants reported normal color vision and normal or corrected-to-normal vision. Power analyses were conducted to establish an appropriate sample size. Effect size for the interaction between color repetition and color-change probability $\left(\eta_{p}^{2}=.50\right)$ and the main effect of color repetition $\left(\eta_{p}^{2}=.84\right)$ were drawn from a comparable study reported in the literature (Thomson, D'Ascenzo, \& Milliken, 2013). These analyses revealed that a sample size of 16 participants was sufficient to detect both the interaction and simple main effects with a power greater than .95 (all Cohen's $f>1$ ). We increased the sample to 20 participants to ensure we had enough power given differences in the analyses conducted here.

Apparatus and stimuli Stimuli were presented using PsychoPy v3.1.5 on a LED monitor with a 144-Hz refresh rate. All displays were presented on a black background that had a luminance value of $.23 \mathrm{~cd} / \mathrm{m}^{2}$. The red and green diamond stimuli were

\footnotetext{
${ }^{1}$ Note that the color-change probabilities here differed from those of Maljkovic and Nakayama (1994; Experiment 2) - the color-change probabilities here were $0, .25, .50, .75$, and 1 , while Maljkovic and Nakayama used the probabilities $0, .50, .70, .90$, and 1 for two participants and $.10, .50, .70, .90$, and 1 for the other participant. We used these probabilities to best assess whether there was a linear relationship between RTs and the probability of a target color change, while also ensuring an equal number of target color repeats and switches across the experimental session.
}

close to isoluminant. Red had a luminance value of $23.63 \mathrm{~cd} /$ $\mathrm{m}^{2}$ and CIE coordinates of .627/.341. Green had a luminance value of $23.56 \mathrm{~cd} / \mathrm{m}^{2}$ and CIE coordinates of .305/.620. All diamonds subtended an approximate vertical and horizontal visual angle of $1^{\circ}$ with a $.17^{\circ}$ cut-off on either the left or the right side. The diamonds were displayed in 12 possible locations distributed evenly on an invisible circle that had a radius of $5^{\circ}$ of visual angle that was centered on a central fixation cross. The white fixation cross subtended a vertical and horizontal visual angle of $.30^{\circ}$ and had a luminance value of $98.11 \mathrm{~cd} / \mathrm{m}^{2} .2$

Procedure Participants' head position was stabilized by a chin and head rest that was positioned $57 \mathrm{~cm}$ from the monitor. Each trial began with a black display with a central fixation cross. The duration of this display was randomized such that it lasted 2-2.5 s. The search display was then displayed, which contained an oddball colored diamond (i.e., the target) and two homogenously colored diamonds (i.e., the distractors). Each of the target and distractor diamonds were randomly assigned to one of the 12 possible locations around fixation. Participants were instructed to locate the target diamond then indicate whether the corner was cut off on the left or right side. Participants indicated a left response by pressing the ' $\mathrm{z}$ ' key and a right response by pressing the ' $\mathrm{m}$ ' key on a standard QWERTY keyboard. The cut-off sides were randomized such that left- and right-side responses were equally likely. The experimental procedure is depicted in Fig. 1.

Each participant performed five blocks that contained 150 trials each (750 trials total). The blocks differed in the probability that the target and distractor colors repeated or switched across trials. The specific target color-change probabilities were: $0, .25$, $.50, .75$, and 1 . Target color-change probabilities were implemented on a trial-by-trial basis across the block of trials. The percentage likelihood of a target color change was displayed on-screen at the beginning of each block, at which time participants were given the opportunity to take a break. The counterbalancing of block order was randomized for each participant such that all block orders were equally likely. Participants performed five practice trials at the beginning of the experimental session to ensure they understood how to perform the search task.

\section{Results}

Correct response times (RTs) less than $200 \mathrm{~ms}$ and greater than 2,000 ms were first removed from analysis, which led to the removal of $2.6 \%$ of observations. The remaining correct RTs were submitted to a non-recursive outlier elimination procedure (Van Selst \& Jolicoeur, 1994), which ensured that different

\footnotetext{
${ }^{2}$ Note that all stimuli in the present experiments were more luminant than those reported by Maljkovic and Nakayama (1994) in their experiment: red $\left(1.98 \mathrm{~cd} / \mathrm{m}^{2}\right)$, green $\left(2.08 \mathrm{~cd} / \mathrm{m}^{2}\right)$, and white $\left(13.2 \mathrm{~cd} / \mathrm{m}^{2}\right)$.
} 


\section{Exp. 1: Color Change Probability: $0, .25, .50, .75,1$}

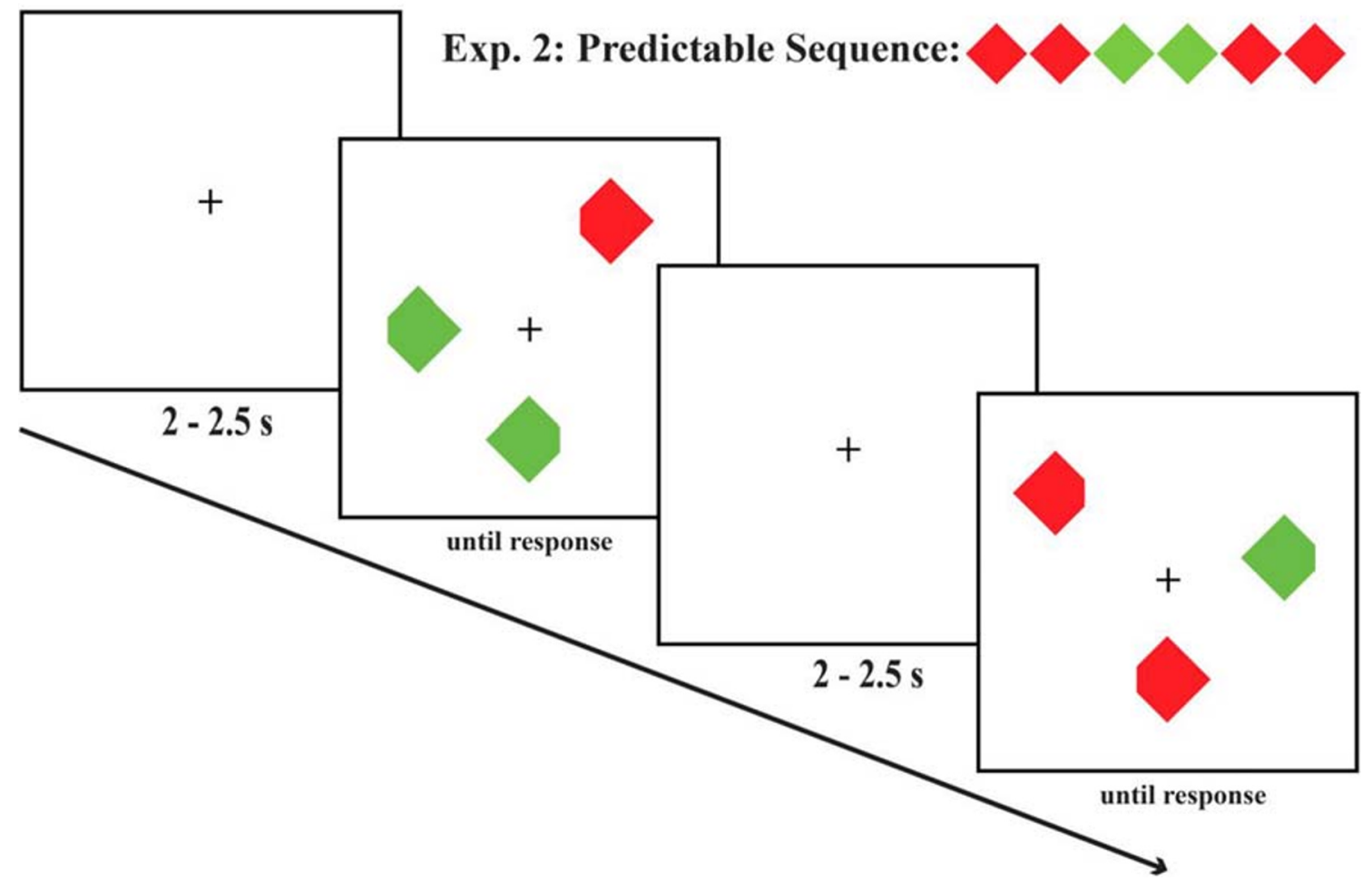

Fig. 1 The diagram depicts the search displays for trials in which the target and distractor colors switched. In Experiment 1, the probability of a color change was varied across blocks. In Experiment 2, the target colors followed a predictable pattern over the course of the passive and active blocks of trials

proportions of observations were not systematically excluded for cells with different numbers of observations. This led to the removal of $2.7 \%$ of observations. Mean RTs and error rates were computed from the remaining observations. An alpha criterion of .05 was used to determine statistical significance. ${ }^{3}$

\footnotetext{
${ }^{3}$ In additional analyses we evaluated whether color-change probability affected both stimulus and response repetitions. These analyses treated response repetition (repeat/switch), color repetition (repeat/switch), and color-change probability block $(.25 / .50 / .75)$ as within-subject factors. This analysis revealed a significant main effect of color repetition, $F(1,19)=52.2, p<.001, \eta_{\mathrm{p}}^{2}=.73$, and response repetition, $F(1,19)=9.59, p=.005, \eta_{\mathrm{p}}^{2}=.34$. The main effect of color repetition reflected shorter RTs when the target color was repeated across trials than when it switched, and the main effect of response repetition reflected shorter RTs when the responses switched across trials than repeated. These main effects were qualified by a three-way interaction that approached significance, $F(2,38)=3.16, p=.054, \eta_{\mathrm{p}}^{2}=.14$. We explored this interaction further by conducting analyses that treated color (repeat/switch) and response (repeat/ switch) repetitions as factors for each color-change probability block. These analyses revealed a non-significant interaction of color and response repetition in the $.50(p=.65)$ and $.75(p=.83)$ color-change probability blocks. There was a significant interaction of color and response repetition in the .25 colorchange probability block, $F(1,19)=9.50, p=.006, \eta^{2}=.33$. This interaction was driven by shorter RTs when both target color and responses switched ( 819 $\mathrm{ms})$ than when the responses switched and the color repeated $(874 \mathrm{~ms})$, $F(1,19)=4.73, p=.04, d=.04$. There were no differences when the responses repeated $(744 \mathrm{~ms})$ or switched $(750 \mathrm{~ms})$ when the color repeated across trials $(p$ $=.64)$. This result supports the notion that the PoP effect constitutes both stimulus- and response-based components (Lamy, Yashar, \& Ruderman, 2010; Lamy, Zivony, \& Yashar, 2011; Töllner, Gramann, Müller, Kiss, \& Eimer, 2008), and that the negative repetition effects produced by the response-based component are modulated by the probability of a color change.
}

Probability analysis To directly test if knowledge of the upcoming target color influences performance, separate one-way within-subject ANOVAs of RTs and error rates were conducted that treated color-change probability $(0 / .25 / .50 / .75 / 1)$ as a factor. The analysis of RTs revealed a significant main effect of color-change probability, $F(4,76)=5.13, p=.001, \eta_{\mathrm{p}}^{2}=.21$. The mean RTs for each of the color-change probability blocks were the following: 0 (654 ms), .25 (796 ms), .50 (792 ms), .75 (803 $\mathrm{ms})$, and $1(811 \mathrm{~ms})$. Given an a priori interest in the influence of expectancy, we conducted separate analyses comparing the 0 and 1 blocks of color-change probability to all other blocks. The analyses revealed responses in the 0 color-change probability block were significantly faster compared to all other blocks (all $F>12.9, p<.002, d\rangle$ .94). ${ }^{4}$ The analyses of the 1 color-change probability block revealed no significant RT differences compared with the $.75, .50$, and .25 blocks (all $F<.3, p>.60$ ). Mean RTs are depicted in Fig. 2.

The analysis of error rates revealed an effect of colorchange probability that approached significance $(p=.08)-$ see the following section for more detail. Mean percentage error rates are depicted in Table 1.

\footnotetext{
${ }^{4}$ Cohen's $d$ values reported in this manuscript were based on aggregate RTs in each condition for each participant.
} 




Fig. 2 Mean response times across color-change probability blocks of Experiment 1. Error bars represent the standard error of the mean corrected to remove between-subject variability (Cousineau, 2005; Morey, 2008)

Color-repetition analysis To further test if the knowledge of the upcoming target color influences performance, separate two-way within-subject ANOVAs were conducted on mean RTs and error rates that treated the three color-change probability blocks $(.25 /$ $.50 / .75$ ) and color repetition (repeat/switch) as within-subject factors. The analysis of RTs revealed a significant main effect of color repetition, $F(1,19)=60.6, p<.001, \eta_{\mathrm{p}}^{2}=.76$, reflecting faster responding for target color repeats than switches. This main effect was qualified by a significant interaction of colorchange probability and color repetition, $F(2,38)=5.16, p=.01$, $\eta_{\mathrm{p}}^{2}=.21$. This interaction was explored further by conducting two additional $2 \times 2$ analyses that treated color-repetition (repeat/ switch) and color-change probability blocks $(.25 / .50 ; .50 / .75)$ as within-subject factors. The analyses comparing the .50 and .75 blocks revealed a significant interaction of color-change probability and color repetition, $F(1,19)=5.89, p=.03, \eta_{p}^{2}=.24$, reflecting that the effect of color repetition was attenuated in the .75 block relative to the .50 block. The analysis comparing the .25 and .50 blocks revealed a non-significant interaction of colorchange probability and color repetition $(p=.46)$. Mean RTs are depicted in Fig. 3.

The analysis of error rates revealed a significant main effect of color repetition, $F(1,19)=5.54, p=.03, \eta_{\mathrm{p}}^{2}=.23$, reflecting higher error rates for target color switches $(1.79 \%)$ than color repeats $(1.28 \%)$. There was also a significant main effect of block, $F(2,38)=4.57, p=.02, \eta_{\mathrm{p}}^{2}=.19$, reflecting higher error rates in the .75 color-change probability block $(2.06 \%)$ relative to the $.50(1.42 \%)$ and $.25(1.14 \%)$ blocks. The interaction of color-change probability and color repetition was not significant $(p=.25)$.

Table 1 Mean error percentages (\%) for color repetition of the colorchange probability blocks of Experiment 1

\begin{tabular}{lccccc}
\hline & 0 & .25 & .50 & .75 & 1 \\
\hline Repeat & 1.67 & 1.43 & 1.27 & 1.15 & - \\
Switch & - & 2.69 & 1.56 & 1.12 & 1.48 \\
\hline
\end{tabular}

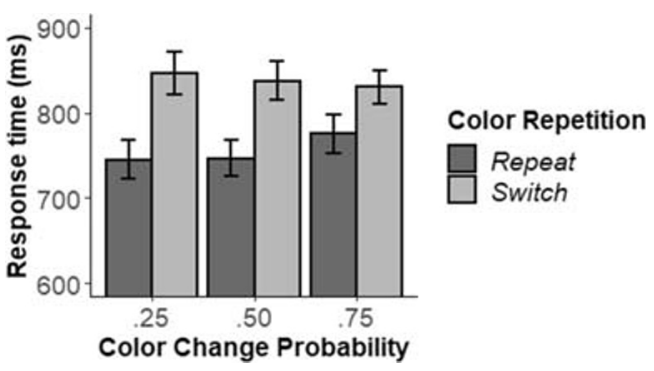

Fig. 3 Mean response times of color repetition for the $.25, .50$, and .75 color-change probability blocks of Experiment 1 . Error bars represent the standard error of the mean corrected to remove between-subject variability (Cousineau, 2005; Morey, 2008)

\section{Discussion}

The purpose of the present experiment was to conceptually replicate Experiment 2 of Maljkovic and Nakayama (1994) in order to determine if the PoP effect can be influenced by the knowledge of the probability of an upcoming target color. Participants performed a color singleton search task where the likelihood of target color repeats and switches varied across blocks of trials. It was revealed that search performance was most efficient when the target color predictably repeated across trials of the search task. This result corresponded well with the findings of Maljkovic and Nakayama. However, it was also revealed that there was similar overall search speed in any block when a target color change occurred. This finding contrasts with that reported by Maljkovic and Nakayama that RTs should become steadily longer with a greater proportion of target color switches. Although it was noteworthy that the PoP effect was robust across all color-change probability blocks, the analyses also revealed that the magnitude of the PoP effect was attenuated by the increased likelihood of a target color switch (see also Thomson, D'Ascenzo, \& Milliken, 2013). Both of these results suggest that the PoP effects in the present experiment were not simply due to the memory representations of the previous target and distractors.

\section{Experiment 2}

With failing to observe the same results as Maljkovic and Nakayama's second experiment, we now conduct a conceptual replication of their fourth experiment where they concluded that an active conscious expectancy of the upcoming target color did not influence the PoP effect. The experimental procedures were essentially the same as the original experiment but now 24 naïve participants performed counter-balanced blocks of singleton searches where the target color could follow a perfectly predictable pattern (e.g., red, red, green, green, red, red, etc.). In the passive block of trials, participants simply performed the search task with the knowledge of the predictable target color sequence. In the active block of trials, 
participants anticipated the upcoming target color by vocalizing its color prior to the onset of the search display. Participants also performed a baseline block of trials where the target color varied unpredictably trial-totrial. ${ }^{5}$ If the PoP effect is attenuated in the active block relative to the baseline and passive blocks, it may suggest that the PoP effect is impacted by an active conscious expectancy. In contrast, if the magnitude of the PoP effect is identical across blocks, it will suggest that it is unaffected by conscious expectancy.

\section{Method}

Participants Twenty-four undergraduates at the University of Toronto participated in exchange for course credit (20 female, $\mathrm{M}_{\text {age }}=18.1$ years). All participants reported normal color vision and normal or corrected-to-normal vision. The sample size was based on the power analyses of Experiment 1; however, four participants were added in order to fully counterbalance block order across participants.

Apparatus and stimuli Apparatus and stimuli were identical to Experiment 1.

Procedure Participants performed a color singleton search task like that of Experiment 1. Participants performed passive, active, and baseline blocks of 240 trials (720 trials total). Block order was fully counterbalanced across participants. In the passive block, the target color followed a predictable pattern (e.g., red, red, green, green, red, red, etc.) and participants were made aware of this pattern. In the active block, the target color followed a predictable pattern like that of the passive block; however, participants were instructed to anticipate the upcoming target color by saying it aloud during the temporal interval prior to the search display onset. In the baseline block, target colors were equally likely, and participants were made aware that they would be unable to predict the target color. Each block began with four practice trials at which time the block specific instructions were delivered. Participants were given the opportunity to take a break at the halfway point and the beginning of each block. An experimenter remained in the room with participants during the total experimental session to ensure compliance during the active block of trials.

\footnotetext{
${ }^{5}$ There were two noteworthy differences in Experiment 2 relative to that of Maljkovic and Nakayama (1994; Experiment 4). First, participants were required to vocalize the upcoming target color rather than subvocalize it. Second, we conducted an additional block of trials where the target color varied unpredictably (i.e., the baseline block). This was done to assess whether the simple knowledge of the target color sequence in the passive condition influenced the PoP effect.
}

\section{Results}

Correct RTs less than $200 \mathrm{~ms}$ and greater than 2,000 ms were first removed from analysis, which led to the removal of $1.5 \%$ of observations. The remaining correct RTs were submitted to a non-recursive outlier elimination procedure (Van Selst \& Jolicoeur, 1994), which led to the removal of $2.8 \%$ of observations. Mean RTs were computed from the remaining observations, and these mean RTs and corresponding error rates were submitted to two-way within-subject ANOVAs that treated color repetition (repeat/switch) and predictability (active/passive/baseline) as within-subject factors. An alpha criterion of .05 was used to determine statistical significance. Mean RTs are depicted in Fig. $4 .^{6}$

The analysis of RTs revealed significant main effects of color repetition, $F(1,23)=73.0, p<.001, \eta_{\mathrm{p}}^{2}=.76$, and predictability, $F(2,46)=3.97, p=.03, \eta_{\mathrm{p}}^{2}=.15$. The main effects were qualified by a significant interaction of color repetition and predictability, $F(2,46)=7.46, p=.002, \eta_{\mathrm{p}}^{2}=.24$. This interaction was explored further by conducting separate analyses comparing each of the predictability blocks.

Analysis of the active and passive blocks revealed a significant interaction of color repetition and predictability, $F(1,23)$ $=12.2, p=.002, \eta_{\mathrm{p}}^{2}=.45$. The main effect of predictability was not significant $(p=.11)$. A separate analysis of color repetition for the active block revealed a significant effect, $F(1,23)=18.3, p<.001, d=.21$, reflecting faster responses for target color repeats $(638 \mathrm{~ms})$ than switches $(677 \mathrm{~ms})$. The analysis of color repetition for the passive block revealed a significant effect, $F(1,23)=73.5, p<.001, d=.60$, reflecting faster responses for target color repeats $(686 \mathrm{~ms})$ than switches (771 ms). The interaction of color repetition and predictability therefore reflects the attenuation of the PoP effect in the active ( $39 \mathrm{~ms}$ ) relative to the passive $(85 \mathrm{~ms})$ block of trials.

Similarly, the analysis of the active and baseline blocks revealed a significant interaction of color repetition and predictability, $F(1,23)=7.41, p=.01, \eta_{\mathrm{p}}^{2}=.24$. The analysis also revealed a main effect of predictability, $F(1,23)=6.58, p=$

\footnotetext{
${ }^{6}$ We conducted an additional analysis that treated response repetition (repeat/ switch), color repetition (repeat/switch), and color predictability block (active/ passive/baseline) as within-subject factors. This analysis revealed a significant main effect of color repetition, $F(1,23)=70.5, p<.001, \eta_{\mathrm{p}}^{2}=.75$, color predictability block, $F(2,46)=4.10, p=.02, \eta_{p}^{2}=.15$, and an effect of response repetition that approached significance $(p=.08)$. Although there was not a significant three-way interaction of color repetition, response repetition, and color predictability block ( $p=.37$ ), we did observe a robust twoway interaction of color and response repetition, $F(1,23)=12.2, p=.002, \eta_{\mathrm{p}}^{2}$ $=.34$. For the target color switch trials, further analyses revealed a significant effect of response repetition, $F(1,23)=10.5, p=.004, d=.19$, reflecting faster responses when responses switched (736 ms) than repeated (758) across trials. For the target color repeat trials, there was no significant difference between whether responses repeated $(679 \mathrm{~ms})$ or switched $(686 \mathrm{~ms})$ across trials $(p=$ .30). Once again, the results support that the PoP effect constitutes stimulusand response-based components (Lamy, Yashar, \& Ruderman, 2010; Lamy, Zivony, \& Yashar, 2011; Töllner et al., 2008), but that the influence of these components did not differ across the color predictability conditions.
} 


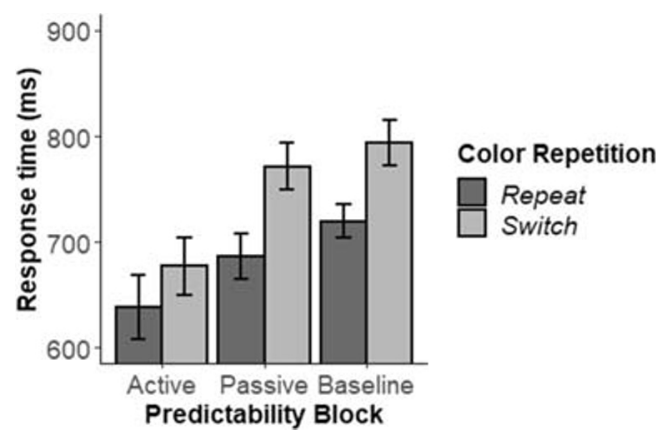

Fig. 4 Mean response times of color repetition for the active, passive, and baseline predictability blocks of Experiment 2. Error bars represent the standard error of the mean corrected to remove between-subject variability (Cousineau, 2005; Morey, 2008)

$.02, \eta_{\mathrm{p}}^{2}=.22$, reflecting overall faster responses in the active block relative to the baseline block. A separate analysis of color repetition for the baseline block revealed a significant effect of color repetition, $F(1,23)=35.8, p<.001, d$ $=.71$, reflecting faster responses for target color repeats $(720 \mathrm{~ms})$ than switches $(793 \mathrm{~ms})$. Once again, the significant interaction of color repetition and predictability indicated that the PoP effect was attenuated in the active (39 $\mathrm{ms})$ relative to the baseline $(73 \mathrm{~ms})$ block of trials.

Analysis of the passive and baseline blocks did not reveal a significant interaction of color repetition and predictability $(p=.35)$ or a main effect of predictability $(p=.27)$.

Analysis of error rates revealed a significant main effect of color repetition, $F(1,23)=8.36, p=.008, \eta_{p}^{2}=$ .27 , reflecting higher error rates for target color switches $(2.26 \%)$ than repeats $(1.70 \%)$. The main effect of predictability $(p=.10)$ and the interaction of color repetition and predictability ( $p=.77$ ) were not significant. Mean percentage error rates are depicted in Table 2.

\section{Discussion}

The purpose of Experiment 2 was to conceptually replicate Maljkovic and Nakayama's (1994) fourth experiment, which investigated the influence of an active conscious expectancy on the PoP effect. It was revealed that the passive and baseline blocks of trials produce PoP effects of similar magnitude, suggesting that the simple knowledge of the upcoming target color did not influence performance. Importantly, when participants were required to vocalize the upcoming target color, the PoP effect was

Table 2 Mean error percentages (\%) for color repetition of the predictability blocks of Experiment 2

\begin{tabular}{lccc}
\hline & Active & Passive & Baseline \\
\hline Repeat & 2.22 & 1.49 & 1.34 \\
Switch & 2.67 & 2.29 & 1.83 \\
\hline
\end{tabular}

significantly attenuated. While the PoP effect was once again robust, this finding directly contrasts the findings of Maljkovic and Nakayama, suggesting instead that an active conscious expectancy can have a profound impact on the PoP effect.

\section{General discussion}

The purpose of the present study was to re-examine two key conclusions of Maljkovic and Nakayama's (1994) seminal paper on the PoP effect. These are: (1) that the knowledge of the probability of a target color change does not influence the PoP effect (Experiment 2), and (2) that an active conscious expectancy of the upcoming target color does not influence the PoP effect (Experiment 4). Here, we conceptually replicated each of these experiments with improved experimental procedures (i.e., appropriate participant numbers, counterbalanced blocks, etc.). In contrast with Maljkovic and Nakayama's second experiment, Experiment 1 of the present study revealed that responses were only fast when the target color was repeated across the entire block of trials - that is, the presence of any color change in a block resulted in comparable overall performance. This pattern of results was in stark contrast with Maljkovic and Nakayama's proposal that responses slowed in a linear manner with the increase in the proportion of target and distractor color switches. Further, the present study demonstrated that the magnitude of the PoP effect decreased as the proportion of target and distractor colors switches increased. In contrast to Maljkovic and Nakayama's fourth experiment, Experiment 2 of the present study revealed that vocalizing the upcoming target color attenuated the PoP effect, demonstrating that the active conscious expectancy condition had a profound influence on performance. Overall, the present study demonstrated that the PoP effect is robust but did not exclusively constitute the automatic priming of the previous target and distractor colors.

\section{Probability knowledge of a color change}

It was observed in both Experiment 1 of the present study and Experiment 2 of Maljkovic and Nakayama (1994) that when the target and distractor colors repeated on every trial in a block, search performance was fast relative to when the target and distractor colors could switch across trials in a block. The interpretation for this finding put forth by Maljkovic and Nakayama was that this search speed benefit occurred since every trial primed the trial before it and that this priming built up over consecutive color repeats. This interpretation, however, cannot be the only reason for the change in search performance given the nonlinear pattern of results across the colorchange probability blocks. One possible reason for this pattern of results was that varying the proportion of repeat and switch 
trials changed how participants performed the search task. When participants were required to find the oddball color target that could unpredictably switch colors with the distractors, they had to prepare for targets that could appear in either color (i.e., oddball targets). However, if the target color remained the same across trials, it was not necessary to prepare for an oddball color, but to prepare for the specific color assigned to the target (e.g., red targets). It may be that target uncertainty necessitates the additional processing step of resolving the target identity. In other words, when the target identity remained the same across trials in a block, participants more quickly determined that the target was indeed the target. Accordingly, we suspect that specific knowledge of the upcoming target color did not benefit search performance but performing the task under conditions when a target color change could occur changed target certainty.

What accounts for the different pattern of results found by Maljkovic and Nakayama (1994; Experiment 2) and the present study? We strongly suspect that it may be due to their participants. Of the three participants in Experiment 2, two were the authors and the third was a naïve participant. Evaluating the pattern of results for each participant, Ken Nakayama's data fits perfectly with the interpretation espoused by their manuscript - that RTs should increase linearly with an increase in the proportion of target color changes in a block. Vera Maljkovic's data fits this same linear pattern somewhat, and the results of the naïve participant fit best with the results here - with the exception of the 1 color-change probability block, where responses were remarkably slower than the $.50, .70$, and .90 color-change probability blocks. The naive participants of Experiments 2 and 4 went by the initials of Z. H. and G. P., respectively. Interestingly, Z. H. and G. P. share initials with Dr. Zijiang He and Dr. Gordon Plant, who published peer-reviewed manuscripts with Ken Nakayama around the same time the paper by Maljkovic and Nakayama (1994) was published. Although these participants may have been naïve to the specific intentions of the experimenters, it is hard to determine whether their exposure to visual search research influenced performance. It is possible that they may have been well practiced at performing visual search tasks and had more developed predictive capabilities compared to typical naive participants, both of which qualities could have changed the distribution of RTs.

Similar to the differences in the RT distributions across color probability blocks noted at the beginning of this section, probability knowledge can also be evaluated by assessing whether the color-change probabilities influenced the magnitude of the PoP effect. In Experiment 1 we conducted analyses that evaluated the magnitude of the PoP effect across $.25, .50$, and .75 color-change probability blocks. These analyses revealed that the magnitude of the PoP effect was significantly larger in the .25 color-change probability block compared to the .50 , and .75 color-change probability blocks. One possibility is that this magnitude difference was due to the explicit knowledge of the color-change probabilities - that is, participants used this knowledge to anticipate the target color. Another possibility is that these magnitude differences were due to differences in the likelihood of multiple consecutive target color repetitions.

One consequence of the probability manipulation used in both the present study and Maljkovic and Nakayama's Experiment 2 was that it invariantly increases not only the likelihood of target and distractor color repetitions from the previous trial, but also the likelihood that the target color could continuously repeat across a number of trials. This is a particularly important issue given that it has been demonstrated that the PoP effect can build up across consecutive target color repeats (Maljkovic \& Martini, 2005; Maljkovic \& Nakayama, 1994). We investigated this issue here by conducting an additional analysis that evaluated only trials that followed two consecutive trial type repetitions (repeat/ switch) for the $.25, .50$, and .75 color-change probability blocks. This analysis did not reveal a significant interaction $(p=.81)$ of color repetition (repeat/switch) and color-change probability block, reflecting that the magnitude of the PoP effect was similar across the $.25(94 \mathrm{~ms}), .50(91 \mathrm{~ms})$, and $.75(77 \mathrm{~ms})$ color-change probability blocks when we controlled for multiple consecutive target color repetitions. Although this result strongly suggests that differences in the magnitude of the PoP effect were influenced by the proportion of multiple consecutive target color repeats, it does not conclusively show that probability knowledge did not also have an impact. For one, this analysis was underpowered and there was a numerical trend indicating that the magnitude of the PoP effect decreased as the probability of a target color change increased. For another, we do not know how blockwide probability knowledge is affected by recent search trials. For example, it is possible that disconfirming information (e.g., a target color repetition when a color switch was probable) influenced whether participants used this probability knowledge. Ultimately, the additional analyses here demonstrated that the build-up of priming across consecutive target color repeats was primarily responsible for the modulation of the magnitude of the PoP effect in Experiment 1, and while this analysis further supports the notion that explicit knowledge of the target color probabilities did not influence performance, more research is required before we can be certain.

\section{Active and passive conscious expectancies}

In Experiment 2 of the present study, it was revealed that vocalizing the upcoming target color significantly attenuated the PoP effect. This finding directly contrasts with Experiment 4 of Maljkovic and Nakayama (1994). The reason why an active conscious expectancy impacted the PoP effect here and not in Maljkovic and Nakayama may have to do with the type of conscious expectancy formed. In Experiment 4 
of Maljkovic and Nakayama, the instruction was to "subvocally utter" the color of the target on the upcoming trial. For pragmatic reasons, in the present study participants were instructed to say the upcoming target color aloud. It has been recently demonstrated that in order to influence a visual search task the type of conscious expectancy matters. While it has been demonstrated that conscious expectancies that are visual in nature can have a profound impact on visual search (Baier \& Ansorge, 2019; Cochrane, Siddhpuria, \& Milliken, 2019; Moriya, 2018), evidence that semantic representations can do so is mixed (Lupyan \& Spivey, 2010; Walenchok, Hout, \& Goldinger, 2016; cf. Baier \& Ansorge, 2019). The inconsistent influence of semantic representations may have to do with the specific parameters of the task. For example, we recently demonstrated that vocalizing a color had a profound impact on color repetition effects, while passive observation of the same symbolic representations did not (Cochrane \& Milliken, 2019). We further demonstrated that whether or not semantic color representations influenced a task depended on the underlying processes (Cochrane \& Milliken, 2020). In other words, the difference between the present findings and those of Maljkovic and Nakayama may be due to subtle differences in the task - it may be that verbal anticipation of the upcoming target color influenced the processes underlying the PoP effect and subvocal utterances did not.

It is worth noting that the attenuation of the PoP effect observed in the active condition of Experiment 2 may have occurred because participants had to perform a secondary task and not because they were forming an active conscious expectancy of the upcoming color. While the present study does a poor job of teasing apart this issue, other studies have demonstrated that an active conscious expectancy can impact the PoP effect. For example, in a recent study we had participants imagine a color that was opposite to that of the previous target color, putting the processes underlying an active conscious expectancy and the PoP effect in opposition (Cochrane, Nwabuike, Thomson, \& Milliken, 2018; see also Cochrane, Zhu, \& Milliken, 2018). Under these conditions the PoP effect gave way to faster search performance when the target color switched (i.e., color imagery congruent trials) than when it repeated (i.e., color imagery incongruent trials). Further, we examined the magnitude of these color imagery effects under conditions when and when not the PoP effect was put in opposition. When participants reported that their color imagery was strong, we observed statistically identical color imagery congruency effects, suggesting that imagery-based active conscious expectancies influence the representations driving the PoP effect.

An unexpected finding in the present study was that informing participants of the predictable target color sequence did not attenuate the PoP effect. This was surprising given the recent findings of Shurygina et al. (2019), which showed that informing participants of the predictable target color sequence prior to a block of color singleton search trials influenced the PoP effect relative to a semi-predictable target color sequence block. We suspect that the different findings here were due to participants not using the predictable target color sequence information. That is, the knowledge of the target color sequence is only useful if participants track and prepare for the upcoming target color. A possible reason Shurygina et al. had better task engagement may be because all blocks of this study provided valid information about the predictability of the upcoming target color. This could have led to greater use of the task instructions since participants were forming an expectancy throughout the entire experimental session. It is also possible that participants were forming an expectancy for some trials in a sequence and not others for the semi-predictable sequence block, which led to the lengthening of RTs. The observed differences between the predictable and semipredictable target color sequence blocks therefore may not be due to a benefit of predictability, but a cost when the target colors were semi-predictable. Overall, we support the conclusion of Shurygina et al. that the knowledge of the upcoming target color can impact the PoP effect, but we suspect it depends on the willingness of participants to use this information.

\section{Conclusions}

The present study clearly reveals that the knowledge of the probability of an upcoming target color switch (Experiment 1) and actively anticipating the upcoming target color (Experiment 2) can have a profound impact on the PoP effect. These findings indicate that the notion that the PoP effect simply reflects the automatic priming of the previous target and distractor colors does not appear to be tenable.

Acknowledgements Financial support for this study was provided in part by a Natural Science and Engineering Research Council of Canada Discovery Grant 2016-06359 awarded to Jay Pratt. The funding agreement ensured the authors' independence in designing the study, interpreting the data, writing, and publishing the report. The authors report no conflict of interest.

\section{References}

Baier, D., \& Ansorge, U. (2019). Investigate the role of verbal templates in contingent capture by color. Attention, Perception, \& Psychophysics, 81(6), 1846-1879.

Bravo, M. J., \& Nakayama, K. (1992). The role of attention in different visual-search tasks. Perception \& Psychophysics, 51(5), 465-472.

Cochrane, B. A., \& Milliken, B. (2019). Imagined event files: an interplay between imagined and perceived objects. Psychonomic Bulletin \& Review, 26(2), 538-544.

Cochrane, B. A., \& Milliken, B. (2020). The representational basis of positive and negative repetition effects. Journal of Experimental Psychology: Human Perception and Performance, 46(3), 252-263. 
Cochrane, B. A., Nwabuike, A. A., Thomson, D. R., \& Milliken, B. (2018). An imagery-induced reversal of intertrial priming in visual search. Journal of Experimental Psychology: Learning, Memory, and Cognition, 44(4), 572-587.

Cochrane, B. A., Siddhpuria, S., \& Milliken, B. (2019). Cueing color imagery: a critical analysis of imagery-perception congruency effects. Journal of Experimental Psychology: Learning, Memory, and Cognition, 45(8), 1410-1421.

Cochrane, B. A., Zhu, H., \& Milliken, B. (2018). Strategic visual imagery and automatic priming effects in pop-out visual search. Consciousness and Cognition, 65, 59-70.

Cousineau, D. (2005). Confidence intervals in within-subject designs: a simpler solution to Loftus and Masson's method. Tutorials in Quantitative Methods for Psychology, 1(1), 42-45.

Lamy, D., Yashar, A., \& Ruderman, L. (2010). A dual-stage account of inter-trial priming effects. Vision Research, 50(14), 1396-1401.

Lamy, D., Zivony, A., \& Yashar, A. (2011). The role of search difficulty in intertrial featuring priming. Vision Research, 51(19), 2099-2109.

Lupyan, G., \& Spivey, M. J. (2010). Redundant spoken labels facilitate perception of multiple items. Attention, Perception, \& Psychophysics, 72(8), 2236-2253.

Maljkovic, V., \& Martini, P. (2005). Implicit short-term memory and event frequency effects in visual search. Visual Research, 45(21), 2831-2846.

Maljkovic, V., \& Nakayama, K. (1994). Priming of pop-out: I. Role of features. Memory \& Cognition, 22(6), 657-672.

Morey, R. D. (2008). Confidence intervals from Normalized data: a correction to Cousineau (2005). Tutorial in Quantitative Methods for Psychology, 4(2), 61-64.

Moriya, J. (2018). Visual mental imagery influences attentional guidance in a visual-search task. Attention, Perception, \& Psychophysics, 80(5), 1127-1142.
Shurygina, O., Kristjánsson, Á., Tudge, L., \& Chetverikov, A. (2019). Expectations and perceptual priming in a visual search task: from eye movements and behavior. Journal of Experimental Psychology: Human Perception and Performance, 45(4), 489-499.

Thomson, D. R., D’Ascenzo, M., \& Milliken, B. (2013). Learning what to expect: context-specific control over intertrial priming effects in singleton search. Memory \& Cognition, 41(4), 522-546.

Töllner, T., Gramann, K., Müller, H. J., Kiss, M., \& Eimer, M. (2008). Electrophysiological markers of visual dimension changes and response changes. Journal of Experimental Psychology: Human Perception and Performance, 34(3), 531-542.

Van Selst, M., \& Jolicoeur, P. (1994). A solution to the effect of sample size on outlier elimination. The Quarterly Journal of Experimental Psychology, 47A(3), 631-650.

Walenchok, S. C., Hout, M. C., \& Goldinger, S. D. (2016). Implicit object naming in visual search: evidence from phonological competition. Attention, Perception, \& Psychophysics, 78(8), 2633-2654.

Open Practices Statement The experiments reported in this article were not preregistered. The data for all experiments are publicly available at the Center of Open Science website (osf.io/683fd). Requests for materials can be sent via email to the corresponding author at brett.cochrane@utoronto.ca.

Publisher's note Springer Nature remains neutral with regard to jurisdictional claims in published maps and institutional affiliations. 